\title{
Identification of novel genes associated with fracture healing in osteoporosis induced by Krm2 overexpression or Lrp5 deficiency
}

\author{
FENG GAO $^{1}$, FENG XU ${ }^{2}$, DANKAI WU ${ }^{1}$, JIEPING CHENG $^{1}$ and PENG XIA ${ }^{1}$ \\ ${ }^{1}$ Department of Orthopedics, The Second Hospital of Jilin University, Changchun, Jilin 130041; \\ ${ }^{2}$ Department of Spine Surgery, The First Hospital of Jilin University, Changchun, Jilin 130021, P.R. China
}

Received January 20, 2016; Accepted January 30, 2017

DOI: $10.3892 / \mathrm{mmr} .2017 .6544$

\begin{abstract}
The aim of the present study was to screen potential key genes associated with osteoporotic fracture healing. The microarray data from the Gene Expression Omnibus database accession number GSE51686, were downloaded and used to identify differentially expressed genes (DEGs) in fracture callus tissue samples obtained from the femora of type I collagen (Collal)-kringle containing transmembrane protein 2 (Krm2) mice and low density lipoprotein receptor-related protein $5^{-1-}$ $\left(\mathrm{Lrp5}^{-/-}\right)$transgenic mice of osteoporosis compared with those in wild-type (WT) mice. Enrichment analysis was performed to reveal the DEG function. In addition, protein-protein interactions (PPIs) of DEGs were analyzed using the Search Tool for the Retrieval of Interacting Genes database. The coexpression associations between hub genes in the PPI network were investigated, and a coexpression network was constructed. A total
\end{abstract}

Correspondence to: Dr Feng Xu, Department of Spine Surgery, The First Hospital of Jilin University, 71 Xinmin Street, Chaoyang, Changchun, Jilin 130021, P.R. China

E-mail: xufengtx1978@163.com

Dr Dankai Wu, Department of Orthopedics, The Second Hospital of Jilin University, 218 Zi Qiang Street, Changchun, Jilin 130041, P.R. China

E-mail:wudankai@163.com

Abbreviations: DEGs, differentially expressed genes; PPIs, protein-protein interactions; BMP-2, bone morphogenetic protein-2; PTH, parathyroid hormone; LRP5, lipoprotein receptor-related protein 5; KREMEN2, kringle containing transmembrane protein 2; DKK1, dickkopf homolog 1; GEO, Gene Expression Omnibus database; RMA, robust microarray analysis; FDR, false discovery rate; GO, Gene Ontology; KEGG, Kyoto Encyclopedia of Genes and Genomes; DAVID, Database for Annotation, Visualization and Integrated Discovery; STRING, Search Tool for the Retrieval of Interacting Genes; PCC, pearson correlation coefficient; CAMs, cell adhesion molecules; $T h b s 2$, thrombospondin 2; $S d c 2$, syndecan 2; Fkbp10, FK506 Binding Protein 10

Key words: fracture healing, osteoporosis, gene, network, coexpression of 841 DEGs (335 upregulated and 506 downregulated) were identified in the Collal-Krm2 vs. the WT group, and 50 DEGs (16 upregulated and 34 downregulated) were identified in the $\mathrm{Lrp5}^{-/-}$vs. the WT group. The DEGs in Collal-Krm 2 mice were primarily associated with immunity and cell adhesion (GO: 0007155) functions. By contrast, the DEGs in $\mathrm{Lrp5}^{-1-}$ mice were significantly associated with muscle system process (GO: 0003012) and regulation of transcription (GO: 0006355). In addition, a series of DEGs demonstrated a higher score in the PPI network, and were observed to be coexpressed in the coexpression network, and included thrombospondin 2 (Thbs2), syndecan 2 ( $S d c 2)$, FK506 binding protein 10 (FkbplO), 2'-5'-oligoadneylate synthase-like protein 2 (Oasl2), interferon induced protein with tetratricopeptide repeats (Ifit) 1 and Ifit 2 . Thbs 2 and $S d c 2$ were significantly correlated with extracellular matrix-receptor interactions. The results suggest that Thbs2, Sdc2, Fkbp10, Oasl2, Ifit1 and Ifit2 may serve important roles during the fracture healing process in osteoporosis. In addition, this is the first study to demonstrate that Sdc2, FkbplO, Oasl2, Ifitl and Ifit 2 may be associated with osteoporotic fracture healing.

\section{Introduction}

Osteoporotic fracture is a common event in the elderly, resulting in substantial mortality, and the mortality rate of hip fracture for 6 months is $\sim 10-20 \%$ (1). The prevalence of osteoporotic fractures, hip fractures in particular, is increasing in many regions of the world (2). Current therapies focus on the prevention and treatment of osteoporotic fractures; however, this may easily lead to complications, thus it remains a worldwide public health concern. Therefore, a greater understanding of the underlying molecular mechanisms of fracture healing in the osteoporotic bone is required, as well as identifying candidate biomarkers for osteoporotic fracture therapies.

Over the past few years, a number of remarkable achievements have been made in the genetic study of fracture healing in osteoporosis. One such study demonstrated that transgenesis of bone morphogenetic protein-2 promotes fracture healing in osteoporosis by inducing increased callus density and a larger cross-sectional callus area (3). During remodeling of fractured bone, parathyroid hormone $(\mathrm{PTH})$ promotes the formation of osteoclasts 
to restore the mechanical strength and structure of bones, and polymorphisms in genes encoding PTH influence the genetic regulation of bone mineral density (4). Low density lipoprotein receptor-related protein 5 (LRP5) serves a significant functional role in skeletal homeostasis, and mutations in LRP5 induce a variety of bone density-associated diseases (5). Lrp5 deficiency results in decreased osteoblast proliferation and function, which induces a low bone mass phenotype (6). Kringle containing transmembrane protein 2 (KREMEN2), also known as KRM2, is a high-affinity transmembrane receptor of dickkopf homolog 1 , and is thought to be a regulator of bone remodeling (7). It has been demonstrated that $\mathrm{Krm}^{-/-}$mice develop a high bone mass phenotype and overexpression of $\mathrm{Krm} 2$ in type I collagen (Colla1)-Krm2 transgenic mice induces severe osteoporosis with decreased levels of osteoblasts and elevated osteoclast differentiation (8). Using a model of fracture healing in Collal-Krm 2 transgenic mice and $\mathrm{Lrp5}^{-/-}$mice, a previous study revealed that fracture healing is greatly damaged in Collal-Krm 2 transgenic mice and $\mathrm{Lrp5}^{-/-}$mice; however, the Collal-Krm2 mice were more severely impaired than $\mathrm{Lrp5}^{-/-}$mice (9). In addition, this previous study identified a set of differentially expressed genes (DEGs) in the two mouse models using microarray analysis (9). However, DEG interactions and functions require further investigation in order to provide a more comprehensive understanding of the effect of osteoporosis on fracture healing.

In order to investigate the interactions and functions of DEGs in Collal-Krm 2 transgenic mice and $\mathrm{Lrp5}^{-/-}$mice further, the microarray data obtained by Liedert et al (9) were analyzed in the present study. Following identification of DEGs, enrichment analysis was performed. In addition, protein-protein interactions (PPIs) of DEGs and hub genes in the PPI network were analyzed. Furthermore, coexpression associations between hub genes and additional DEGs were examined. These results may contribute to a greater understanding of the effect of osteoporosis on fracture healing, and provide novel information that facilitates the development of future clinical therapies for osteoporotic fractures.

\section{Materials and methods}

Affymetrix microarray data. The raw gene expression profile dataset GSE51686 (9) was obtained from the Gene Expression Omnibus (GEO) database (http://www.ncbi.nlm. nih.gov/geo/). The data was generated by the (Mouse430_2) Affymetrix Mouse Genome 430 2.0 Array platform (GEO accession, GPL1261; Affymetrix, Inc., Santa Clara, CA, USA). This dataset contained 9 fracture callus tissue samples obtained from the femora at 10 days following osteotomy in the wild-type (WT) mice ( $\mathrm{n}=3$ ), Colla1-Krm 2 transgenic mice with severe osteoporosis ( $\mathrm{n}=3$ ), and $\mathrm{Lrp5}^{-/-}$mice with low bone mass $(n=3)$, respectively. All mice were female and 26 weeks of age.

The CEL and probe annotation files for this dataset were downloaded. The raw expression data were preprocessed by background correction, quantile normalization and probe summarization using the robust microarray analysis algorithm in the affy package (version 3.3.2) (10) of Bioconductor (version 3.4; http://www.bioconductor.org/). Subsequently, the
org.Hs.eg.db (version 3.4.0) (11) and illuminaHumanv3.db (version 1.26.0) (12) packages of Bioconductor were used to translate probe identifications (IDs) to gene symbols. If one gene symbol was matched by multiple probe IDs, the mean expression value was selected as the expression level of this gene.

Identification of DEGs. DEGs in Collal-Krm2 mice and $\mathrm{Lrp5}^{-/}$mice compared with the WT controls were identified using the linear models for microarray data (LIMMA) package (version 3.30.3; http://www.bioconductor.org/packages/release/bioc/html/limma.html) (13), which is a commonly used tool for the identification of DEGs. The P-value for each gene was calculated using the unpaired t-test in LIMMA, which was then adjusted for the false discovery rate (FDR) using the Benjamini-Hochberg method (14). Only the genes with FDR values $<0.05$ and $\log _{2}$ fold change values $\geq 0.5$ were selected as DEGs.

The Venny online tool (version 2.0; http://bioinfogp.cnb. csic.es/tools/venny/index.html) (15) was utilized to construct Venn diagrams for the upregulated and downregulated genes identified between the Collal-Krm 2 vs. WT and $L r p 5^{-/}$vs. WT groups.

Enrichment analysis of DEGs. Functional Gene Ontology (GO) and Kyoto Encyclopedia of Genes and Genomes (KEGG) pathway enrichment analyses of upregulated and downregulated genes were performed using the Database for Annotation Visualization and Integrated Discovery (version 6.8; http://david.abcc.ncifcrf.gov/) database (16). The P-value was calculated using the modified Fisher's exact test, and $\mathrm{P}<0.05$ was considered to indicate a statistically significant difference. A gene count in each term $\geq 2$ was set as the cut-off criteria. Additional parameters were set to the default values.

Construction of PPI networks. PPIs of DEGs were obtained from the Search Tool for the Retrieval of Interacting Genes database (version 10.0; http://string-db.org/), which integrates a variety of known and predicted protein associations (17). The combined score for each PPI was calculated, and a score of $>0.4$ was set as the cut-off criterion. Additional parameters were set to the default values. The PPI network was visualized using the Cytoscape software (version 3.4.0; http://cytoscape .org/), which is an open access software for visualizing biomolecular networks (18). In the network, 'node' represents a gene or protein, and 'line' represents an interaction between the two nodes. The degree of each node (number of interactions with other proteins) is equal to the number of nodes that interacted with this node.

Analysis of hub genes in the PPI network. Hub genes refer to the relatively key genes in the network. Hub genes were identified using three centricity methods in the PPI network, including the degree centrality (19), betweenness centrality (20) and subgraph centrality methods (21). The scores obtained from the degree, betweenness and subgraph methods were calculated using the CytoNCA plug-in (version 2.1.6) (22) in Cytoscape. High scores for the degree, betweenness and subgraph methods indicated that the nodes 
A

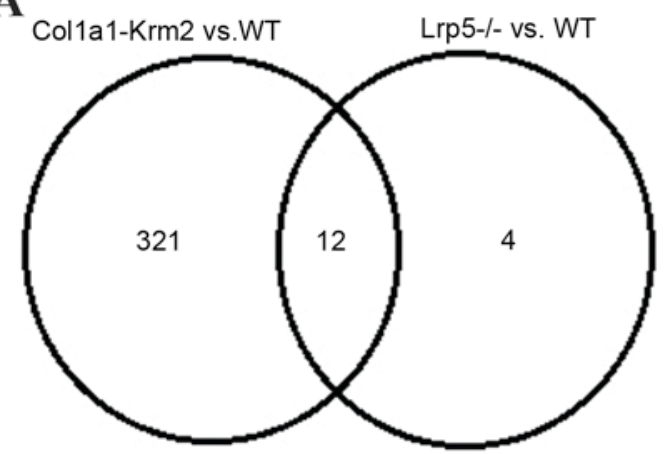

B

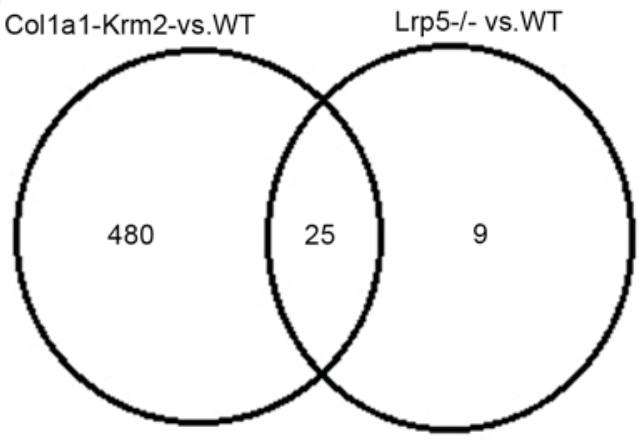

Figure 1. Venn diagrams of differentially expressed genes between Colla1-Krm2 vs. WT and Lrp5 $5^{-/}$vs. WT groups. (A) Venn diagram of upregulated genes. (B) Venn diagram of downregulated genes. Collal, type I collagen; Krm2, kringle containing transmembrane protein 2; Lrp5, low density lipoprotein receptor-related protein 5; Colla1-Krm2, Colla1-Krm2 transgenic mice; Lrp5 ${ }^{-/}$, mice deficient in Lrp5; WT, wild-type mice.

A

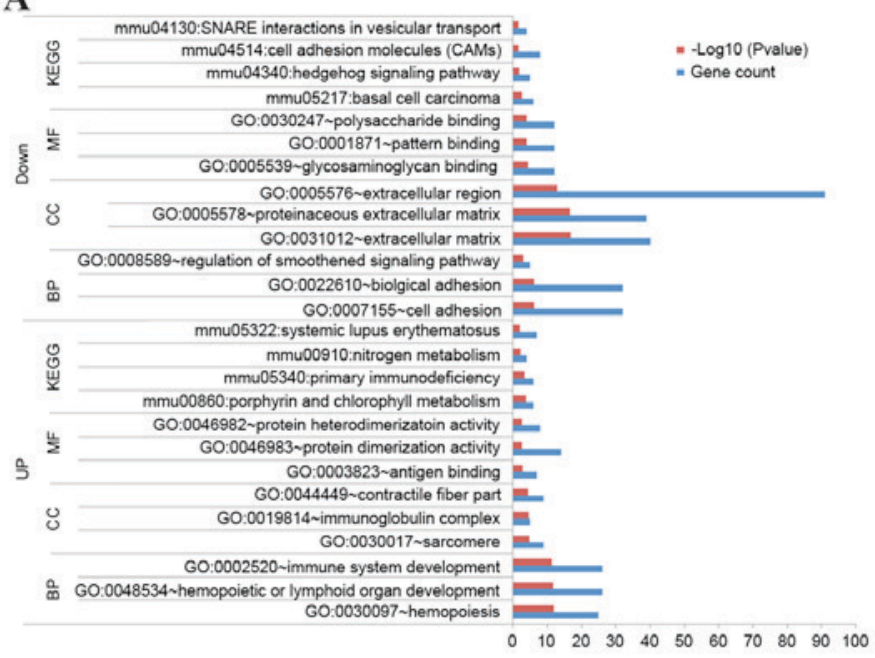

B

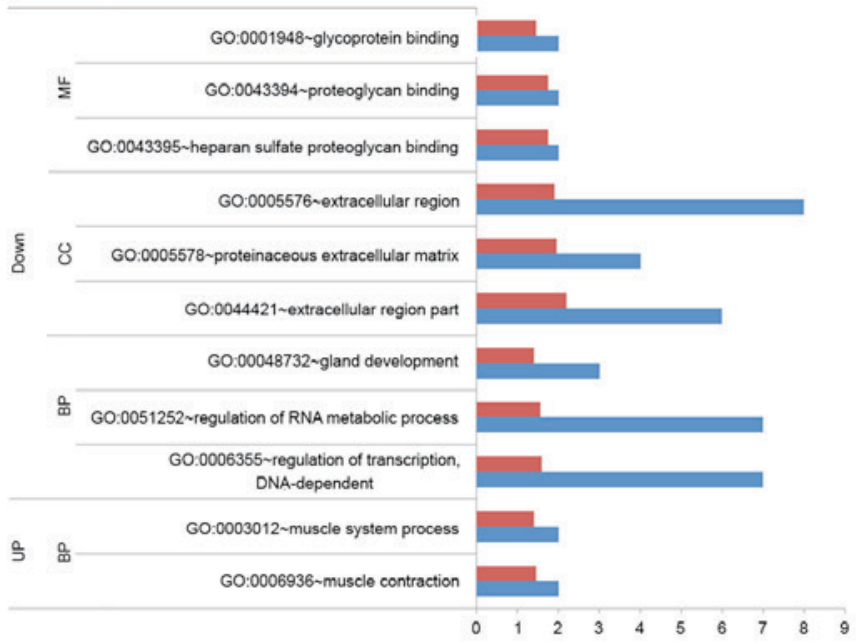

Figure 2. GO and KEGG pathway terms enriched by differentially expressed genes between (A) Colla1-Krm2 transgenic mice and wild-type mice and

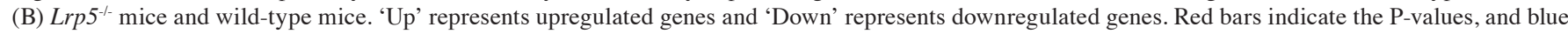
bars indicate the gene count. GO, Gene Ontology; KEGG, Kyoto Encyclopedia of Genes and Genomes; Collal, type I collagen; Krm2, kringle containing transmembrane protein 2; Lrp5, low density lipoprotein receptor-related protein 5; BP, biological process; CC, cellular component; MF, molecular function .

were more significant in the network. Hierarchical clustering of hub genes with higher scores was performed using the pvclust R package (version 1.3-2) (23).

Coexpression associations of hub genes with DEGs. The Pearson's correlation coefficient (PCC) method (24) was used to identify the coexpression associations of hub genes with other DEGs. Only coexpression associations with PCC values of $>0.9$ were selected for analysis. A PCC value of $>0$ indicated that the two genes were positively correlated, and a PCC value of $<0$ indicated that the two genes were negatively correlated.

\section{Results}

Statistical analysis. Based on the cut-off criteria, a total of 841 DEGs (335 upregulated and 506 downregulated) and 50 DEGs (16 upregulated and 34 downregulated) were identified in the Collal-Krm 2 and $\mathrm{Lrp5}^{-/-}$mice when compared with WT mice, respectively. When compared with WT mice, 12 of these genes were upregulated and 25 were downregulated in the Collal-Krm2 and $\mathrm{Lrp5}^{-/-}$mice (Fig. 1).

$D E G$ function. To further reveal gene function in the two groups, GO and KEGG pathway enrichment analyses were performed. In the Collal-Krm 2 vs. WT group, the upregulated genes were primarily associated with hemopoiesis (GO: 0030097), hemopoietic or lymphoid organ (GO: 0048534) and immune system development (GO: 0002520), as well as pathways associated with primary immunodeficiency (mmu05340) and nitrogen metabolism (mmu00910) (Fig. 2A). The downregulated genes were significantly associated with cell adhesion (GO: 0007155) and regulation of the smoothened signaling pathway, as well as the hedgehog signaling pathway (mmu04340) and cell adhesion molecules (mmu04514) (Fig. 2A).

In the $\mathrm{Lrp5}^{-/-}$vs. WT group, the upregulated genes were implicated in muscle contraction (GO: 0006936) and muscle system process (GO: 0003012) (Fig. 2B). The downregulated genes were markedly associated with the regulation of 


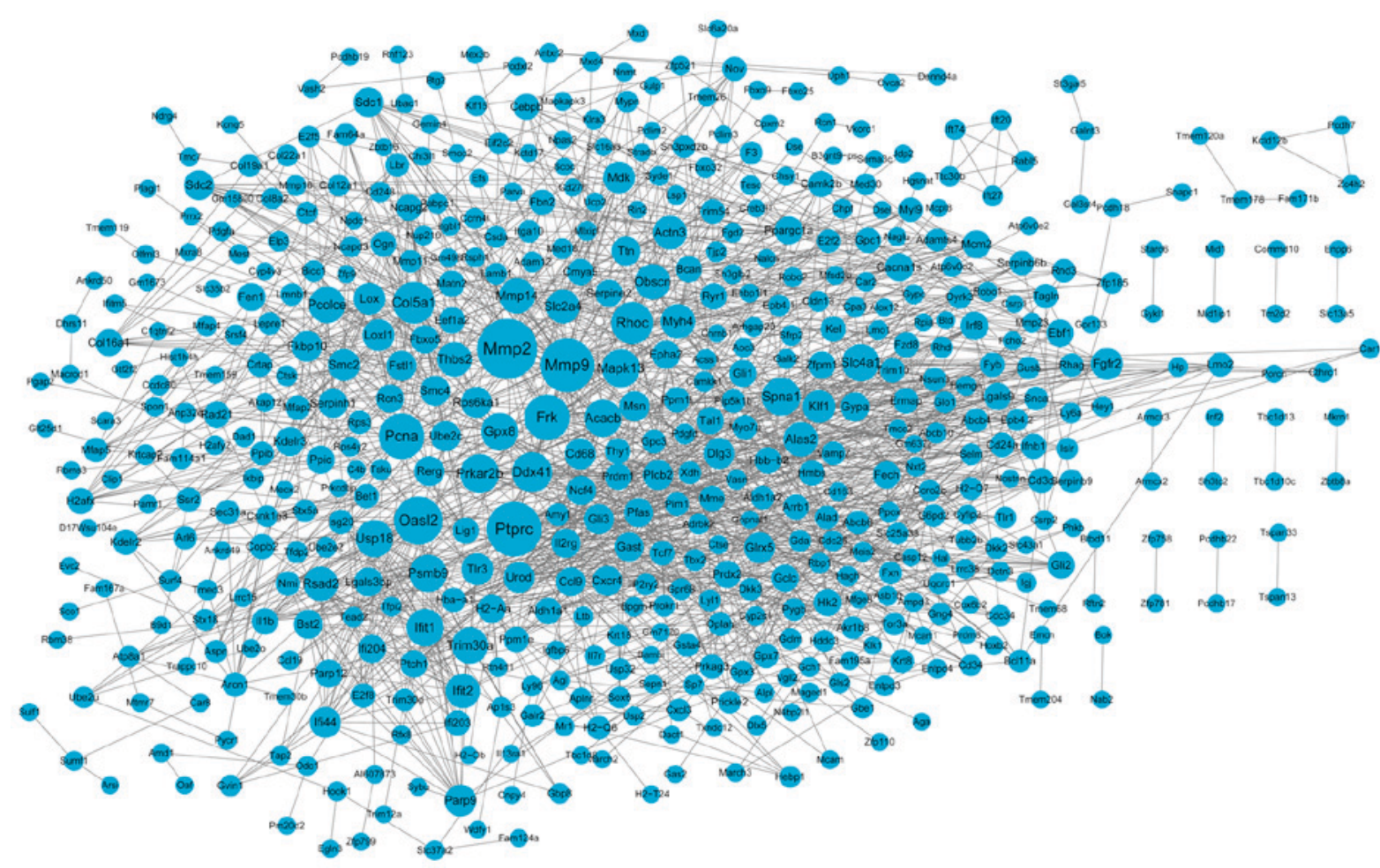

Figure 3. Protein-protein interaction network of differentially expressed genes as determined using the Search Tool for the Retrieval of Interacting Genes database (http://string-db.org/). Each node represents a protein, and each line represents the interaction between the two proteins.

transcription (GO: 0006355) and RNA metabolic processes (GO: 0051252) (Fig. 2B). No significant pathways were enriched by the upregulated genes.

Analysis of PPI network. In order to determine interactions between DEGs, a PPI network was constructed. The network was composed of 551 nodes and 1,608 PPIs (Fig. 3). Based on the centricity methods, the top 40 nodes with the highest scores in the PPI network were selected as hub genes for further analysis, including 2'-5'-oligoadneylate synthase-like protein 2 (Oasl2), thrombospondin 2 (Thbs2), syndecan 2 (Sdc2), FK506 binding protein 10 ( $F k b p 10)$, interferon induced protein with tetratricopeptide repeats (Ifit) 1 and Ifit2 (Table I). Following the removal of duplicates in Table I, a total of 66 genes remained, which were clustered into two groups and used to distinguish the WT, Collal-Krm2 and Lrp $5^{-1}$ samples in a heat map (Fig. 4).

The 66 hub genes were significantly associated to the five signaling pathways (Table II). Matrix metalloproteinase (Mmp) 2 and Mmp9 were associated with the leukocyte transendothelial migration pathway, whereas Thbs2 and $S d c 2$ were associated with the extracellular matrix (ECM)-receptor interaction pathway. The protein tyrosine phosphatase receptor type $\mathrm{C}$ and $\mathrm{Sdc} 2$ were implicated in the cell adhesion molecule pathway (Table II).

Analysis of the coexpression network. In order to investigate the coexpression associations between the selected hub genes and additional DEGs, a coexpression network was constructed. A total of 21 hub genes were determined to coexpress with additional DEGs (Fig. 5). A set of hub genes were observed to coexpress with each other, including Thbs2, $S d c 2$ and Fkbp10, as well as Oasl2, Ifitl and Ifit2 (Fig. 5).
Table I. Top 40 nodes with a high score in the protein-protein interaction network.

\begin{tabular}{lr} 
A, Subgraph & \\
\hline Node & Score \\
\hline Mmp2 & 132118.94 \\
Oas12 & 115994.88 \\
Ifit1 & 95969.49 \\
Trim30a & 92368.98 \\
Mmp9 & 89980.91 \\
Usp18 & 87137.86 \\
Ifit2 & 83711.57 \\
Rsad2 & 78389.78 \\
Parp9 & 65845.10 \\
Psmb9 & 62329.73 \\
Ifi44 & 61890.17 \\
Ptprc & 60581.35 \\
Bst2 & 52788.61 \\
Lgals3bp & 52307.49 \\
Parp12 & 50636.56 \\
Col5a1 & 42741.73 \\
Ifi204 & 32780.95 \\
Thbs2 & 32724.00 \\
Tlr3 & 32063.93 \\
Lgals9 & 30456.22 \\
Pcolce & 27954.06 \\
Mmp14 & 27788.79 \\
Ifi203 & 27352.81 \\
Pcna & 26264.86
\end{tabular}


Table I. Continued.

A, Subgraph

\begin{tabular}{lc}
\hline Node & Score \\
\hline Nmi & 25586.03 \\
Frk & 25365.84 \\
Loxl1 & 24301.15 \\
Lox & 23572.33 \\
Isg20 & 21952.91 \\
Rhoc & 20744.50 \\
Mdk & 20643.03 \\
Fst11 & 20386.14 \\
Ddx41 & 19990.48 \\
Sdc1 & 18627.42 \\
Serpinh1 & 17851.99 \\
Gpx8 & 17771.15 \\
Rcn3 & 17400.74 \\
Cd68 & 17032.79 \\
Sdc2 & 14213.72 \\
Fkbp10 & 14067.95 \\
\hline
\end{tabular}

B, Degree

Mmp2

Ptprc

Mmp9

Oasl2

Pcna

Frk

Col5a1

Rhoc

Ddx41

Prkar2b

Spna1

Mapk13

Ifit 1

Usp18

Psmb9

Gpx8

Acacb

Slc4a1

Trim30a

Pcolce

Mmp14

Obscn

Ifit 2

Rsad2

Thbs 2

Myh4

Alas2

Slc2a4

Actn3

Klf1

Parp9

Tlr3
Table I. Continued.

B, Degree

Node Score

\begin{tabular}{ll}
\hline Lox11 & 16.00 \\
Lox & 16.00 \\
Mdk & 16.00 \\
Fgfr2 & 16.00 \\
Smc2 & 16.00 \\
Ifi44 & 15.00 \\
Cd68 & 15.00 \\
Fkbp10 & 15.00 \\
\hline
\end{tabular}

C, Betweenness

Ptprc

30306.94

Mmp2

Pcna

30018.82

26498.73

24770.62

20782.62

17367.36

16584.40

16291.33

13323.41

13268.12

11615.84

11494.19

11248.73

10606.64

10462.83

9557.37

9042.65

8806.07

8625.42

7343.19

6612.19

6597.27

6507.87

6370.79

6325.04

6084.76

5320.19

5243.41

5180.35

5082.71

5063.36

5019.16

4905.46

4728.94

4708.25

4565.29

4559.99

4274.92

4171.70

3997.35 


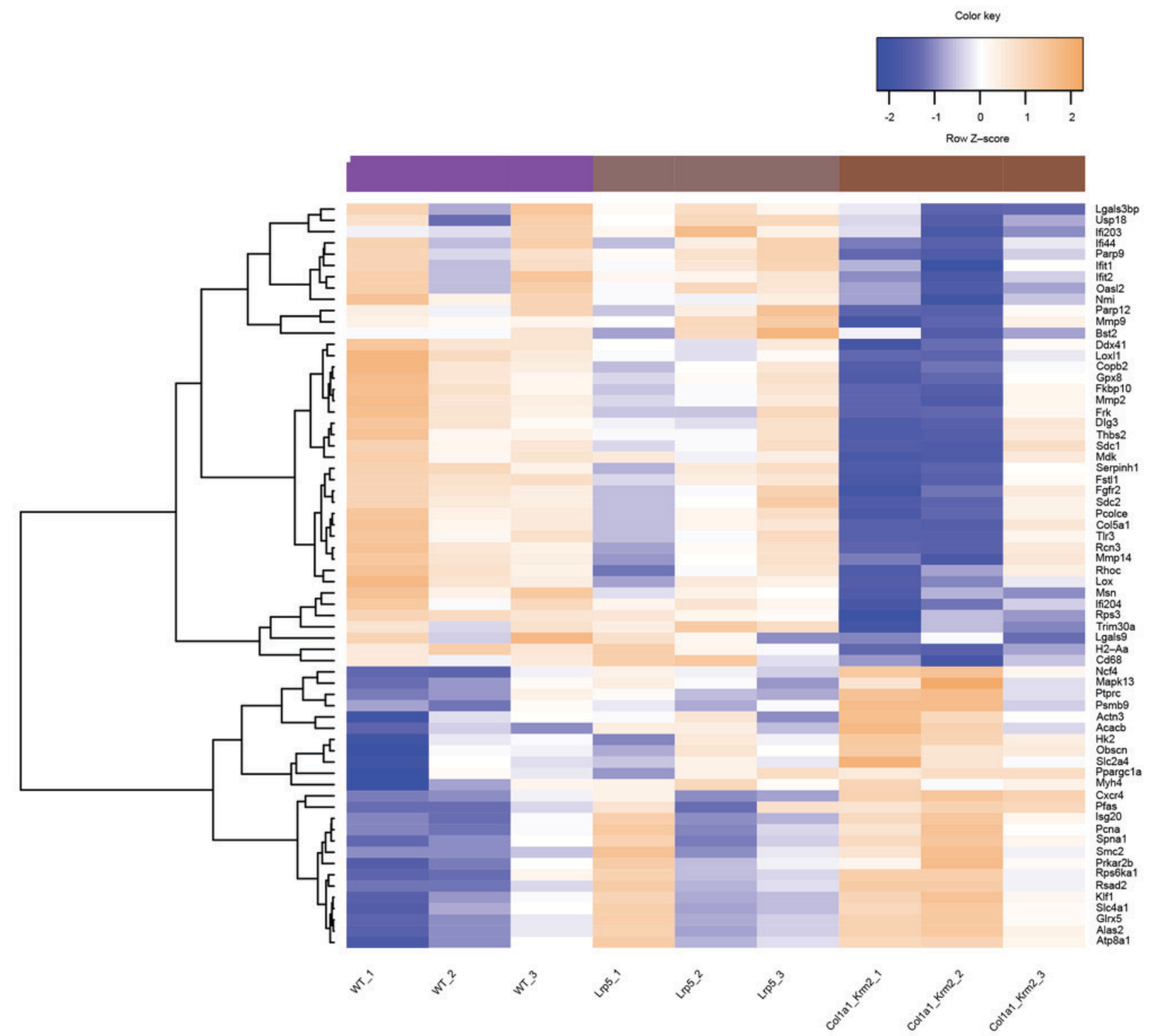

Figure 4. Heat map of hub genes in the protein-protein interaction network in WT, Lrp $5^{-/}$and Collal-Krm 2 mice. Each row represents a single gene and each column represents a sample. The gradual color alteration from orange to blue represents the process from upregulation to downregulation of genes. WT, wild-type; Collal, type I collagen; Krm2, kringle containing transmembrane protein 2; Lrp5, low density lipoprotein receptor-related protein 5.

\section{Discussion}

In the present study, a set of 841 DEGs (335 upregulated and 506 downregulated) and 50 DEGs (16 upregulated and 34 downregulated) were identified in the Collal-Krm2 vs. WT and $\operatorname{Lrp~}^{-/-}$vs. WT groups, respectively. A number of DEGs demonstrated a high score in the PPI network, and were coexpressed in the coexpression network. These genes included Thbs2, Sdc2 and Fkbp10, as well as Oasl2, Ifit1 and Ifit2. Thbs 2 and $S d c 2$ were associated with the ECM-receptor interaction pathway.

Thbs 2 is a part of the thrombospondin family and mediates cell-to-cell and cell-to-matrix interactions (25). A previous review reported that disrupted $T h b s 2$ expression increases cortical bone density, accelerates fracture healing, induces resistance to ovariectomy-induced bone loss and alters the pattern of load-induced bone formation (26). In Thbs2-null mice, marrow-derived osteoprogenitor cells are increased, and endosteal bone formation is promoted, indicating that Thbs 2 modulates the proliferation of osteoprogenitor cells and bone remodeling $(27,28) . S d c 2$ functions as an integral membrane protein and mediates cell-to-matrix interactions via its ECM protein receptor (29). $S d c 2$ is a crucial determinant of chemosensitivity in osteoblasts, and it stimulates the mitogenic activity of granulocyte-macrophage colony-stimulating factor (30). FkbplO is a part of the FKBP-type peptidyl-prolyl cis/trans isomerase family and interacts with collagens (31). A homozygous splicing mutation in FkbplO leads to osteogenesis imperfecta with a mineralization defect via a reduction in bone collagen content $(32,33)$. There is no direct evidence to implicate $S d c 2$ and FkbplO in osteoporotic fracture healing, however, they are thought to coexpress with Thbs 2 . Therefore, $S d c 2$ and $F k b p 10$, as well as Thbs 2 may serve key roles during the fracture healing process in osteoporosis, via their coexpression associations with each other. 
Table II. Pathways enriched by the hub genes in the protein-protein interaction network.

\begin{tabular}{llll}
\hline KEGG entry term: pathway & P-value & Gene count & \\
\hline mmu04670: Leukocyte transendothelial migration & $1.82 \times 10^{-5}$ & 7 & Mapk13, Cxcr4, Mmp9, Ncf4, Msn, Actn3, Mmp2 \\
mmu04910: Insulin signaling pathway & 0.00420 & 5 & Prkar2B, Slc2A4, Hk2, Acacb, Ppargc1A \\
mmu04512: ECM-receptor interaction & 0.00740 & 4 & Sdc1, Thbs2, Col5A1, Sdc2 \\
mmu04514: Cell adhesion molecules & 0.03803 & 4 & Ptprc, Sdc1, H2-Aa, Sdc2 \\
mmu04920: Adipocytokine signaling pathway & 0.04178 & 3 & Slc2A4, Acacb, Ppargc1A
\end{tabular}

KEGG, Kyoto Encyclopedia of Genes and Genomes; ECM, extracellular matrix.

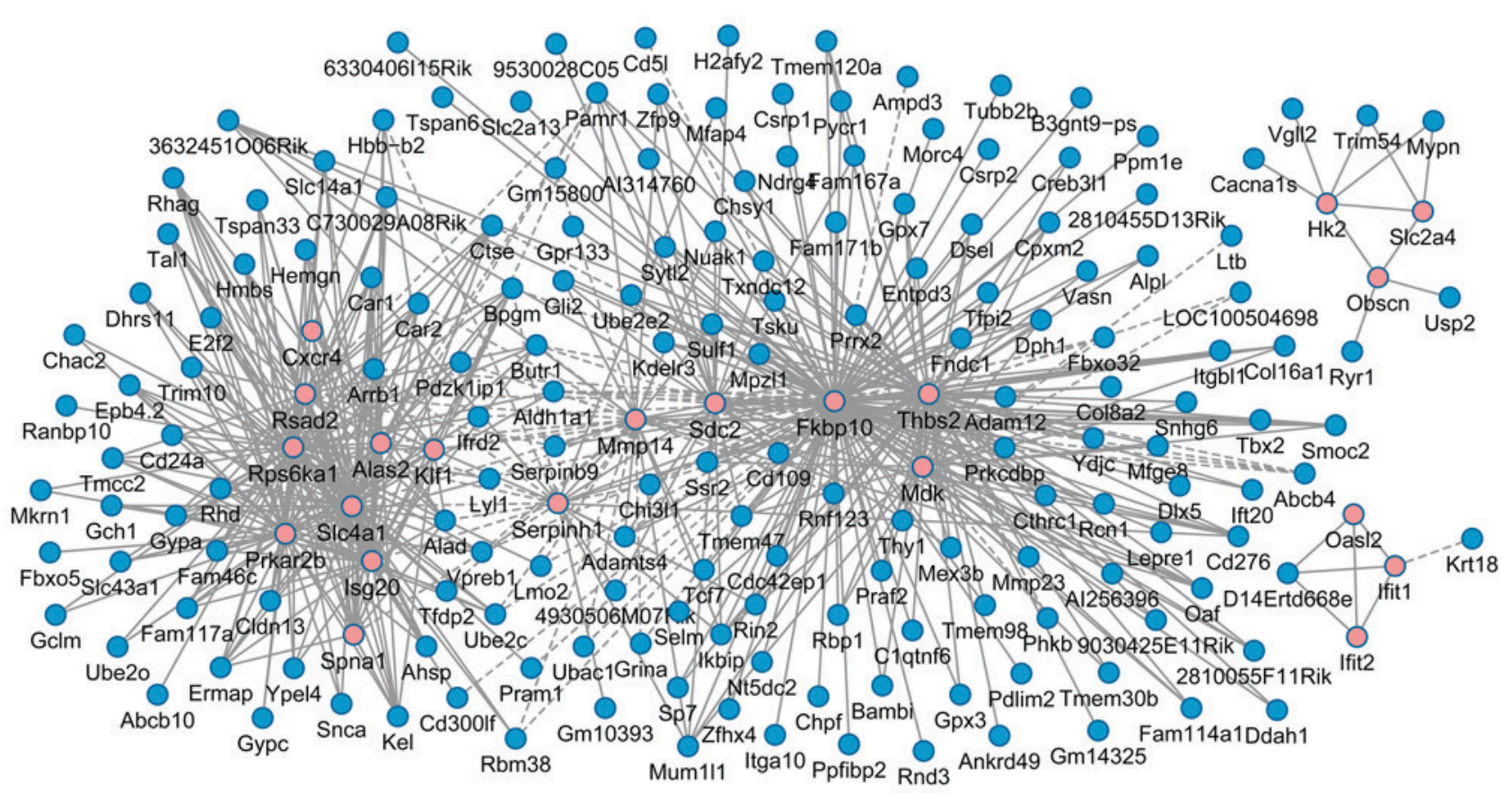

Figure 5. Coexpression network of hub genes and additional differentially expressed genes. Red-colored nodes represent hub genes in the protein-protein interaction network, and blue-colored genes represent differentially expressed genes that are not hub genes. Each node represents a protein, and each line represents the coexpression association between the two genes. Solid lines indicate the coexpression (pearson correlation coefficient) $>0.9$ (positive correlation), and the dotted lines indicate the coexpression (pearson correlation coefficient) $<0.9$ (negative correlation).

In the present study, Oasl2, Ifit 1 and Ifit 2 demonstrated high scores in the PPI network and coexpressed with each other. Ifitl and Ifit2 were interferon-induced proteins containing tetratricopeptide repeats (34). Ifitl is known to be an important innate immune bottleneck (35). During the response of osteoblasts to immune cytokine interferon- $\beta$, the expression of IfitI is induced (36). Ifit 2 and Oasl2 are involved in innate immunity $(37,38)$. Only a limited number of studies have investigated the association between the Ifit1, Ifit2 and Oasl2 genes and fracture repair; however they present potential novel candidates for osteoporotic fracture repair therapies.

In the present study, the number of identified DEGs in the Colla1-Krm2 vs. WT group was markedly higher than that observed in the $\operatorname{Lrp5} 5^{-/}$vs. WT group, which was consistent with previous findings (9). According to the DEGs enrichment analysis, the DEGs in the Colla1-Krm2 vs. WT group were primarily associated with immunity and cell adhesion. By contrast, the DEGs in the $L r p 5^{-1}$ vs. WT group were significantly associated with muscle system processes (GO: 0003012) and the regulation of transcription (GO: 0006355). These results suggest that during the fracture repair process in osteoporosis, the DEGs induced by $\mathrm{Krm} 2$ overexpression or Lrp 5 deficiency, and their functions, may be distinctly different.

Compared with the findings presented by Liedert et al (9), the present study identified the interactions and coexpression patterns among a set of genes, which was not determined previously. However, these predictions require validation in further studies. In a future study, the DEGs and their interactions will be determined in patients.

In conclusion, a series of DEGs, including Thbs $2, S d c 2$ and Fkbp10, as well as Oasl2, Ifitl and Ifit2, demonstrated a significant role in the PPI network and were observed to form co-expression patterns. The results suggest that these genes may serve crucial roles during the fracture repair process in osteoporosis. Sdc2, Fkbp10, Oasl2, Ifit1 and Ifit2 were demonstrated to be novel genes associated with osteoporotic fracture healing. 


\section{References}

1. Namkung-Matthai H, Appleyard R, Jansen J, Hao Lin J, Maastricht S, Swain M, Mason RS, Murrell GA, Diwan AD and Diamond T: Osteoporosis influences the early period of fracture healing in a rat osteoporotic model. Bone 28: 80-86, 2001.

2. Johnell $\mathrm{O}$ and Kanis JA: An estimate of the worldwide prevalence and disability associated with osteoporotic fractures. Osteoporos Int 17: 1726-1733, 2006.

3. Egermann M, Baltzer AW, Adamaszek S, Evans C, Robbins P, Schneider E and Lill CA: Direct adenoviral transfer of bone morphogenetic protein-2 cDNA enhances fracture healing in osteoporotic sheep. Hum Gene Ther 17: 507-517, 2006.

4. Noordin S and Glowacki J: Parathyroid hormone and its receptor gene polymorphisms: Implications in osteoporosis and in fracture healing. Rheumatol Int 36: 1-6, 2016.

5. Mizuguchi T, Furuta I, Watanabe Y, Tsukamoto K, Tomita H, Tsujihata M, Ohta T, Kishino T, Matsumoto N, Minakami H, et al: LRP5, low-density-lipoprotein-receptor-related protein 5, is a determinant for bone mineral density. J Hum Genet 49: 80-86, 2004

6. Kato M, Patel MS, Levasseur R, Lobov I, Chang BH, Glass DA II, Hartmann C, Li L, Hwang TH, Brayton CF, et al: Cbfa1-independent decrease in osteoblast proliferation, osteopenia, and persistent embryonic eye vascularization in mice deficient in Lrp5, a Wnt coreceptor. J Cell Biol 157: 303-314, 2002.

7. Mao B, Wu W, Davidson G, Marhold J, Li M, Mechler BM, Delius H, Hoppe D, Stannek P, Walter C, et al: Kremen proteins are Dickkopf receptors that regulate Wnt/beta-catenin signalling. Nature 417: 664-667, 2002.

8. Schulze J, Seitz S, Saito H, Schneebauer M, Marshall RP Baranowsky A, Busse B, Schilling AF, Friedrich FW, Albers J, et al: Negative regulation of bone formation by the transmembrane Wnt antagonist Kremen-2. PLoS One 5: e10309, 2010.

9. Liedert A, Röntgen V, Schinke T, Benisch P, Ebert R, Jakob F, Klein-Hitpass L, Lennerz JK, Amling $M$ and Ignatius A: Osteoblast-specific Krm2 overexpression and Lrp5 deficiency have different effects on fracture healing in mice. PLoS One 9 e103250, 2014.

10. Gautier L, Cope L, Bolstad BM and Irizarry RA: affy-analysis of Affymetrix GeneChip data at the probe level. Bioinformatics 20 307-315, 2004.

11. Carlson M: org.Hs.eg.db: Genome wide annotation for Human $\mathrm{R}$ package version 3.4.0, 2015.

12. Dunning M, Lynch A and Eldridge M: illuminaHumanv3.db: Illumina HumanHT12v3 annotation data (chip illuminaHumanv3). R package version 1.26.0, 2015.

13. Ritchie ME, Phipson B, Wu D, Hu Y, Law CW, Shi W and Smyth GK: limma powers differential expression analyses for RNA-sequencing and microarray studies. Nucleic Acids Res 43: e47, 2015 .

14. Glueck DH, Mandel J, Karimpour-Fard A, Hunter L and Muller KE: Exact calculations of average power for the Benjamini-Hochberg procedure. Int J Biostat 4: Article 11, 2008.

15. Oliveros JC: VENNY. An interactive tool for comparing lists with Venn Diagrams. 2007. http://bioinfogp.cnb.csic.es/tools/ venny/index.html. Accessed November 20, 2013.

16. Huang DW, Sherman BT, Tan Q, Collins JR, Alvord WG, Roayaei J, Stephens R, Baseler MW, Lane HC and Lempicki RA: The DAVID gene functional classification tool: A novel biological module-centric algorithm to functionally analyze large gene lists. Genome Biol 8: R183, 2007.

17. Szklarczyk D, Franceschini A, Wyder S, Forslund K, Heller D, Huerta-Cepas J, Simonovic M, Roth A, Santos A, Tsafou KP, et al: STRING v10: Protein-protein interaction networks, integrated over the tree of life. Nucleic Acids Res 43 (Database issue) D447-D452, 2015.

18. Kohl M, Wiese S and Warscheid B: Cytoscape: Software for visualization and analysis of biological networks. Methods Mol Biol 696: 291-303, 2011.

19. Jeong H, Mason SP, Barabási AL and Oltvai ZN: Lethality and centrality in protein networks. Nature 411: 41-42, 2001.
20. Goh KI, Oh E, Kahng B and Kim D: Betweenness centrality correlation in social networks. Phys Rev E Stat Nonlin Soft Matter Phys 67: 017101, 2003.

21. Estrada E and Rodriguez-Velázquez JA: Subgraph centrality in complex networks. Phys Rev E Stat Nonlin Soft Matter Phys 71: 056103, 2005.

22. Tang Y, Li M, Wang J, Pan Y and Wu FX: CytoNCA: A cytoscape plugin for centrality analysis and evaluation of protein interaction networks. Biosystems 127: 67-72, 2015.

23. Suzuki R and Shimodaira H: Pvclust: An R package for assessing the uncertainty in hierarchical clustering. Bioinformatics 22: 1540-1542, 2006.

24. Adler J and Parmryd I: Quantifying colocalization by correlation: The Pearson correlation coefficient is superior to the Mander's overlap coefficient. Cytometry A 77: 733-742, 2010.

25. LaBell TL, Milewicz DJ, Disteche CM and Byers PH: Thrombospondin II: Partial cDNA sequence, chromosome location, and expression of a second member of the thrombospondin gene family in humans. Genomics 12: 421-429, 1992.

26. Alford AI and Hankenson KD: Matricellular proteins: Extracellular modulators of bone development, remodeling, and regeneration. Bone 38: 749-757, 2006.

27. Hankens on KD, Bain SD, Kyriakides TR, Smith EA, Goldstein SA and Bornstein P: Increased marrow-derived osteoprogenitor cells and endosteal bone formation in mice lacking thrombospondin 2. J Bone Miner Res 15: 851-862, 2000.

28. Delany AM and Hankenson KD: Thrombospondin-2 and SPARC/osteonectin are critical regulators of bone remodeling. J Cell Commun Signal 3: 227-238, 2009.

29. Ishikawa-Brush Y, Powell JF, Bolton P, Miller AP, Francis F, Willard HF, Lehrach $\mathrm{H}$ and Monaco AP: Autism and multiple exostoses associated with an $\mathrm{X} ; 8$ translocation occurring within the GRPR gene and 3' to the SDC2 gene. Hum Mol Genet 6: 1241-1250, 1997 .

30. Modrowski D, Baslé M, Lomri A and Marie PJ: Syndecan-2 is involved in the mitogenic activity and signaling of granulocyte-macrophage colony-stimulating factor in osteoblasts. J Biol Chem 275: 9178-9185, 2000.

31. Ishikawa Y, Vranka J, Wirz J, Nagata K and Bächinger HP: The rough endoplasmic reticulum-resident FK506-binding protein FKBP65 is a molecular chaperone that interacts with collagens. J Biol Chem 283: 31584-31590, 2008.

32. Venturi G, Monti E, Dalle Carbonare L, Corradi M, Gandini A, Valenti MT, Boner A and Antoniazzi F: A novel splicing mutation in FKBP10 causing osteogenesis imperfecta with a possible mineralization defect. Bone 50: 343-349, 2012.

33. Schwarze U, Cundy T, Pyott SM, Christiansen HE, Hegde MR, Bank RA, Pals G, Ankala A, Conneely K, Seaver L, et al: Mutations in FKBP10, which result in Bruck syndrome and recessive forms of osteogenesis imperfecta, inhibit the hydroxylation of telopeptide lysines in bone collagen. Hum Mol Genet 22: 1-17, 2013.

34. Wathelet MG, Clauss IM, Content J and Huez GA: The IFI-56K and IFI-54K interferon-inducible human genes belong to the same gene family. FEBS Lett 231: 164-171, 1988.

35. McDermott JE, Vartanian KB, Mitchell H, Stevens SL, Sanfilippo A and Stenzel-Poore MP: Identification and validation of Ifit1 as an important innate immune bottleneck. PLoS One 7: e36465, 2012.

36. Woeckel VJ, Eijken M, van de Peppel J, Chiba H, van der Eerden BC and van Leeuwen JP: IFN $\beta$ impairs extracellular matrix formation leading to inhibition of mineralization by effects in the early stage of human osteoblast differentiation. J Cell Physiol 227: 2668-2676, 2012.

37. Perwitasari O, Cho H, Diamond MS and Gale M Jr: Inhibitor of $\kappa \mathrm{B}$ kinase epsilon (IKK(epsilon)), STAT1, and IFIT2 proteins define novel innate immune effector pathway against West Nile virus infection. J Biol Chem 286: 44412-44423, 2011.

38. Zhu J, Ghosh A and Sarkar SN: OASL-a new player in controlling antiviral innate immunity. Curr Opin Virol 12: 15-19, 2015. 